\title{
FRESNEL-AIRY FORMULAS FOR QUASI-ANISOTROPIC MEDIA
}

Mikhail G. Evtikhov

Kotel'nikov Institute of Radioengineering and Electronics of RAS, Fryazino Branch, http://fire. relarn.ru

Fryazino 141190, Moscow region, Russian Federation emg20022002@mail.ru

Abstract. The Fresnel-Airy equations for quasi-anisotropic media are derived. In isotropic and in some anisotropic media, an elliptically polarized plane electromagnetic wave can be represented as two independent waves with linear polarization. Such media admit a generalization of the Fresnel equations and are called quasi-isotropic. The purpose of this paper is to derive the Fresnel-Airy equations that allow solving Fresnel problems for multilayer quasi-anisotropic media plates with arbitrary thicknesses. For example, resonant structures appearing on the angular diagrams of reflection and transmission coefficients for thick layers of isotropic and anisotropic ice covered with a thin layer of water are considered. With such observations, it is sufficient to change the orientation of the recording device and there is no need to change the thickness of the plates. The dielectric constant of ice, orthogonal to the plane of reflection, affects only waves with p-polarization. The dielectric constant of ice parallel to the plane of reflection affects only waves with s-polarization. Resonance effects between the waves in the ice plate and in a thin layer of water are theoretically obtained. In the Appendix, the components of the tensors of complex permittivity and Complex permeability that are admissible for quasi-anisotropic media are discussed. The class of quasi-anisotropic media is much broader than the cases considered in the example, it includes all isotropic media, as well as some ferromagnetic and, possibly, gyrotropic media under certain conditions.

Keywords: reflection, refraction, Fresnel equations, permittivity, millimeter waves, ice, resonance, multilayer plates

УАК 535.391.4, 53.082.53, 53.083.2

Bibliography - 16 references

Received 27.05.2018

RENSIT, 2018, 10(1):91-100

DOI: 10.17725/rensit.2018.10.091

Contents

1. INTRODUCTION (91)

2. ON the parameters that Clarify THE SYMMETRY OF THE FRESNEL EQUATIONS (92)

3. Algorithm for calculating the COEFFICIENTS FOR MULTILAYER PLATES (94)

4. Calculation of the coefficients FOR ICE (95)

5. Conclusion (97)
Appendix 1 (98)

ApPendix 2 (99)

REFERENCES (99)

\section{INTRODUCTION}

The Fresnel equations were first derived in 1823. Applications of the Fresnel equations are manifold $[1,2,3,4]$, they take place also in ellipsometry [5], in remote sensing [6]. It is assumed that the waves are plane and linear, the media are isotropic and homogeneous. Generalizations of 
Fresnel equations to the case of one or several plane-parallel plates are called Fresnel-Airy equations, they are written in terms of Fresnel equations [1-5].

In isotropic linear media, an elliptically polarized monochromatic electromagnetic wave can be represented as two independently propagating linearly polarized waves. Let the wave vector be 0 along the $z$ axis. The structure of the fields of waves can be represented as $\left(H_{x,} H_{y}, E_{2}\right)$ and $\left(E_{x}, E_{y}, H_{2}\right)$. TE-waves (transverse electric) has field structure $\left(H_{\varkappa}, H_{y}, E_{2}\right) ; \quad$ TM-waves (transverse magnetic) has field structure $\left(H_{x}, H_{y}\right.$, E) [1]. If the plane of separation of the media is $x=0$, then the type of the original wave, the reflection wave and the transmission wave are the same. In plane parallel multilayered plates with smooth boundaries, waves of various types propagate independently. In optical terms, TE-waves is $s$-polarize waves; TMwaves is $p$-polarize waves [1-5].

TE waves are also used to describe the propagation of electromagnetic waves in ferromagnetic films with magnetization to saturation. In such anisotropic media, TE waves exist not in all directions, but only in certain distinguished directions $[7,8]$. The requirements on the tensors of the dielectric and magnetic permeability, at which TE- and TMwaves exists, were considered in $[9,10]$ and the corresponding generalizations of the Fresnel equations were obtained. Let quasi-anisotropic media are media that satisfy these requirements, defined and discussed below as (1), (2), see also Appendix 1. The class of quasianisotropic media is quite wide. It is possible to apply generalized Fresnel equations to many Fresnel problems of both magnetic and nonmagnetic media, as well as for remote sensing problems.

The purpose of this paper is to obtain an algorithm for calculating the FresnelAiry coefficients for multilayer planeparallel plates of quasi-anisotropic media with an arbitrary number of layers and arbitrary layer thicknesses.

To achieve this goal, the Fresnel formulas obtained earlier in $[9,10]$ reduce to a more symmetric form of notation and generalize to the case of plane-parallel plates with smooth boundaries using the mathematical apparatus of extremely sparce matrices proposed in $[11,12]$. The derivation of Fresnel's formulas using visual representations and the assumption of medium transparency is not entirely suitable for a medium with absorption. Further, more abstract methods based on the Maxwell equations and on approaches from [1] are used.

\section{ON THE PARAMETERS THAT CLARIFY THE SYMMETRY OF THE FRESNEL EQUATIONS}

In optics, when considering Fresnel problems, the concepts of s-polarization and p-polarization of electromagnetic waves are used [3]. Other terms and methods are also used to represent an elliptically polarized wave as two linearly polarized waves [2]. In remote sensing problems, horizontal and vertical polarizations (with respect to the horizon line) are considered [6]. 
In [1] we used the definition of the concepts of $s$ - and $p$-polarization, somewhat different from the traditional definition. In the case of $s$-polarization, the electric field vector is parallel to the plane of reflection and the wave has the form of a TE wave. In the case of $p$-polarization, the magnetic field vector appears parallel to the plane of reflection and the wave has the form of a TM-wave. In such definitions, The concept of the "plane of incidence" is not used, the TE-, TMwave definitions, are applicable to media with absorption, waves with complex wave vectors in media with complex dielectric and magnetic permeabilities. (It should be noted that for media with amplification, requires methods that take into account causality [13]). The transmission amplitude coefficients for $p$-polarization obtained in [1] differ from the coefficients usually given in the reference books. To agree the records of the formulas it is sufficient to take into account that the amplitude of the $p$-wave in [1] is characterized by the amplitude of the magnetic field. For transparent media, the amplitude of the $p$-wave is reduced to the electric field by the coefficient $\sqrt{\frac{\mu}{\varepsilon}}$, called the "wave resistance" [14]. In the following, we shall use the amplitude of the $p$-wave, determined from magnetic field.

The following corollaries of the Maxwell equation for media without free charges and currents are valid $[9,10]$.

If in both media the following conditions are satisfied: $\varepsilon_{13}=\varepsilon_{23}=0, \mu_{31}=\mu_{32}=0$,

then, for the incident wave with $s$-polarization the reflected and refracted waves remain waves with $s$-polarization.

The wave vector along the $z$-direction is equal to 0 .

The dispersion equation includes only the coefficients $\mu_{11}, \mu_{22}, \mu_{12}, \mu_{21}, \varepsilon_{33}$, the amplitude of the wave with $s$-polarization does not depend on the remaining 9 components.

If in both media the following conditions are satisfied:

$\mu_{13}=\mu_{23}=0, \varepsilon_{31}=\varepsilon_{32}=0$,

then for the incident wave with $p$-polarization the reflected and refracted waves remain waves with $p$-polarization.

The wave vector along the z-direction is equal to 0 .

The dispersion equation includes only the coefficients $\varepsilon_{11}, \varepsilon_{22}, \varepsilon_{12}, \varepsilon_{21}, \mu_{33}$, the amplitude of the wave with $p$-polarization does not depend on the remaining 9 components.

The Maxwell equation for media without free charges and currents are invariant with respect to substitution

$$
\mathbf{E} \rightarrow \mathbf{H} ; \mathbf{H} \rightarrow-\mathbf{E} ; \hat{\boldsymbol{\mu}} \rightarrow \hat{\boldsymbol{\varepsilon}} ; \hat{\boldsymbol{\varepsilon}} \rightarrow \hat{\boldsymbol{\mu}} .
$$

This substitution changes the order of the Maxwell equations, but the system of equations remains unchanged. This substitution transforms a wave with $s$-polarization into a wave with $p$-polarizations. Although the substitution (3) is of the 4th order, but because of the linearity of the system of equations, it manifests itself in the formulas as a 
substitution of the second order. In the formulas with this transformation, dielectric and magnetic permeability should replace each other (see (3)). This fact was used both in [1] and in [9, 10], it was pointed in [2] as a useful technique.

We associate the substitution (3) with the parameter $e$, taking the values -1 and 1. We define the dielectric and magnetic permeability as interrelated functions depending on the parameter $e$, so that $\mu(e=$ 1) $=\mu ; \mu(e=-1)=\varepsilon$. In this notation, after formulas for $s$-polarization are obtained, to obtain formulas for $p$-polarization it will be sufficient to multiply the parameter $e$ by $(-1)$.

In accordance with $[9,10]$ under constraints (1) or (2) in plane-parallel multilayer plates, the components of the complex wave vector $k_{z}$ are equal to 0 , the components of $k_{y}$ are common for all waves. Value $k_{x}$ is solutions of the quadratic dispersion equation. In each layer there are 2 values of $k_{x}$ corresponding to the transmitted and reflected wave. We define the parameter $q$, which denotes the direction of propagation of the wave along the $x$. The parameter $q$ takes the values -1 and 1 and is used to resolve the ambiguity of the solution of the quadratic dispersion equation $k_{x}=k_{x 1} \pm k_{x 0}=k_{x 1}$ $+q k_{x 0^{\circ}}$ Let the harmonic wave pass in the medium with the number $u$ a layer of thickness $d[u]$ along the $x$ axis, then its amplitude is multiplied by the value $\beta_{\mathrm{e}, \mathrm{q},[\mathrm{u}]}$ $=\exp \left(i\left(k_{\mathrm{x}, \mathrm{e}, \mathrm{u}]}\right) q d_{[\mathrm{u}]}\right)$. In isotropic media, $k_{\mathrm{x} 1}$ turns out to be zero, $q^{2}=1$ and the factor $\beta_{\mathrm{e}, \mathrm{q},[\mathrm{n}]}$ does not depend on the parameter $q$. In the general case of quasi-anisotropic media, one must take into account the direction of propagation of the wave. Amplitude coefficients of reflection, transmission, and coefficient $\beta_{\mathrm{e}, \mathrm{q},[\mathrm{n}]}$, obtained by $[9,10]$, taking into account the parameters $e, q$ and the number of the medium $u$, are represented as functions $R(e, q, u), T(e, q, u), \beta(e, q, u)$, and are given in Appendix 2.

\section{ALGORITHM FOR CALCULATING THE COEFFICIENTS FOR MULTILAYER PLATES}

The system of equations for the wave amplitudes in a multilayer plate is represented in a vector-matrix form:

$$
\widehat{\mathbf{U P}}=\mathbf{V} \text {. }
$$

The solution of this linear equation, the vector $P$, is a vector of the FresnelAiry coefficients, it consists of the ratios of the wave amplitudes formed in the multilayer plate to the amplitude of the incident wave. Let $m$ be the number of layers in the plate. The $1 s t$ and $m+2$ semi-infinite media are outside the plate. The number of the incident wave is 0 . The resulting reflected wave has number 1, the total transmitted wave has the number $2 m+2$. Vectors $V, P$ and a square matrix $U$ have $2 m+2$ rows. Each row $u$ of the matrix and the component of the vector $V$ corresponds to the equation for the wave with the number u. All components are assumed to be dependent on the parameter $e$. For $e$ $=1$ the system describes waves with $s$-polarization. Value $e=-1$ coresponds to waves with $p$-polarization. The 
complex amplitudes of the waves with $p$-polarization are given by the magnetic field. The vector $V$ describes the terms of the equations that depend on the amplitude of the incident wave. In the case when the incident wave comes from the first medium, $V_{1}=\mathrm{R}(1, \mathrm{e}, 1), V_{2}=$ $T(1, \mathrm{e}, 1), \ldots$, the remaining components of $V$ are zero. The matrix components fix the interconnection of the waves, taking into account the multiple reflections of the waves in the multilayer plate.

In $[11,12]$, as an alternative to block matrices, it was suggested to use extremely sparse matrices and extensives. In our case, we have a class of square matrices of size $(2 m+2) \times(2 m+2)$. The matrix $S(n$, $k)$ is an extremely sparse matrix of natural arguments $n$ and $k$ if all its components are zero except for one equal to one in the $n$th row and the kth column. The sum $U=\sum^{2 m+2} U_{n, k} S(n, k)$ corresponds to square matrix $\tan ^{n}=k(2 m+2) \times(2 m+2)$, where $U_{n, k}$ are the components of the matrix. If there is some regularity in the matrix, then it can be expressed in terms of a partition of the common sum into partional sums. We will form the matrix as a sum $\hat{\mathbf{U}}=\sum^{m} \mathbf{U}_{n}$ where $n$ is the number of layers of ${ }^{=1}$ the multilayer plate. Equations for the waves on the first and on the last boundaries of the multilayer plate are expressed by the matrix $\mathbf{U}_{1}$. The matrices $\mathbf{U}_{2}, \ldots, \mathbf{U}_{\mathrm{m}}$ describe the waves at the boundaries of the second and third, ..., $m$ th and $m+1$ media.

$$
\begin{aligned}
& \hat{\mathbf{U}}_{1}=\hat{\mathbf{S}}(1,1)-\beta(e,-1,2) T(e,-1,2) \hat{\mathbf{S}}(1,3)+ \\
& +\hat{\mathbf{S}}(2,2)-\beta(e,-1,2) R(e,-1,2) \hat{\mathbf{S}}(2,3)+ \\
& +\hat{\mathbf{S}}(2 m+1,2 m+1)- \\
& -\beta(e, 1, m+1) R(e, 1, m+1) \hat{\mathbf{S}}(2 m+1,2 m)+ \\
& +\hat{\mathbf{S}}(2 m+2,2 m+2)- \\
& -\beta(e, 1, m+1) T(e, 1, m+1) \hat{\mathbf{S}}(2 m+2,2 m) ; \\
& \hat{\mathbf{U}}_{n}=\hat{\mathbf{S}}(2 n-1,2 n-1)- \\
& -\beta(e, 1, n) R(e, 1, n) \hat{\mathbf{S}}(2 n-1,2 n-2)- \\
& -\beta(e,-1, n+1) T(e,-1, n+1) \hat{\mathbf{S}}(2 n-1,2 n+1)+ \\
& +\hat{\mathbf{S}}(2 n, 2 n)-\beta(e, 1, n) T(e, 1, n) \hat{\mathbf{S}}(2 n, 2 n-2)- \\
& -\beta(e,-1, n+1) R(e,-1, n+1) \hat{\mathbf{S}}(2 n, 2 n+1) .
\end{aligned}
$$

\section{CALCULATION OF THE COEFFICIENTS FOR ICE}

To illustrate the new possibilities for solving Fresnel problems using the concepts of quasi-anisotropic media, we construct the simplest example based on the results from [14]. In this work, the angular diagram (dependence on the slip angle) is given for the spectral emissivity of a two-layer plate at a frequency of 1 $\mathrm{GHz}$ for s-polarization. The thickness of each layer is $160 \mathrm{~cm}$. The dielectric permittivities of the layers are close to the properties of ice and water. A resonance structure of the intensity of plane electromagnetic waves consisting of 3 peaks is discussed. Similar resonance oscillations of the intensity in the ice plate were considered in [15] with a change in the thickness of the plate. The idea of observing resonance peaks in angle diagrams (dependencies on the slip angle) is very attractive, such observations do not require changing the thickness of the plate, it is sufficient to change the 
orientation in the space of the recording device. However, this device must have a narrow bandwidth.

Let us consider theoretically at the same frequency $1 \mathrm{GHz}$ an isotropic ice plate with a thickness of $320 \mathrm{~cm}$. Angular diagrams for the FresnelAiry coefficients at $\varepsilon=3.18+\mathrm{i} 0.0007$, (permittivity of ice, for frequency $1 \mathrm{GHz}$ and temperature $0^{\circ} \mathrm{C}$, calculated by the Debye formula [16]) are shown in Fig. 1. Three resonance peaks of the intensity of $s$-polarization waves are observed in accordance with [14]. For $p$-polarization, there are also 3 resonant peaks.

How will the angular diagram change if the permittivity becomes anisotropic and decreases along the $x$
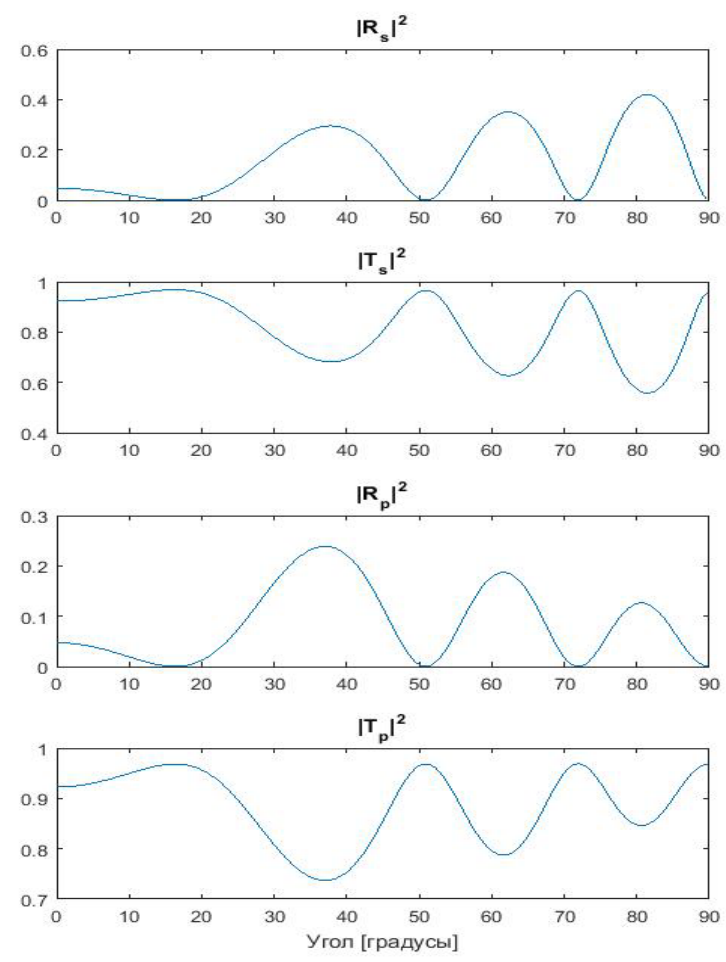

Fig. 1. Angular diagrams of reflection and transmission coefficients of waves with s-and p-polarizations $1 \mathrm{GHz}$, for an isotropic ice plate $320 \mathrm{~cm}$ thick, at $\varepsilon=3.18+$ i0.0007. axis to a value of $\varepsilon_{11}=1.5+\mathrm{i} 0.003$ ? (This value is characteristic for dry snow [16]). Fig. 2 shows that the value of the permittivity along the $x$ axis does not affect waves with $s$-polarization, but the number of resonant peaks of waves with p-polarization increases. The $s$-polarization waves do not depend on $\varepsilon_{11}$ (see Appendix 1). It was checked next facts. An increase in $\varepsilon_{11}$ leads to a decrease in the number of resonance peaks of waves with $p$-polarization. An increase in the imaginary part of $\mathrm{e}_{11}$ leads to damping of these waves. A similar effect is exerted by $\varepsilon_{33}$ on waves with $s$-polarization.
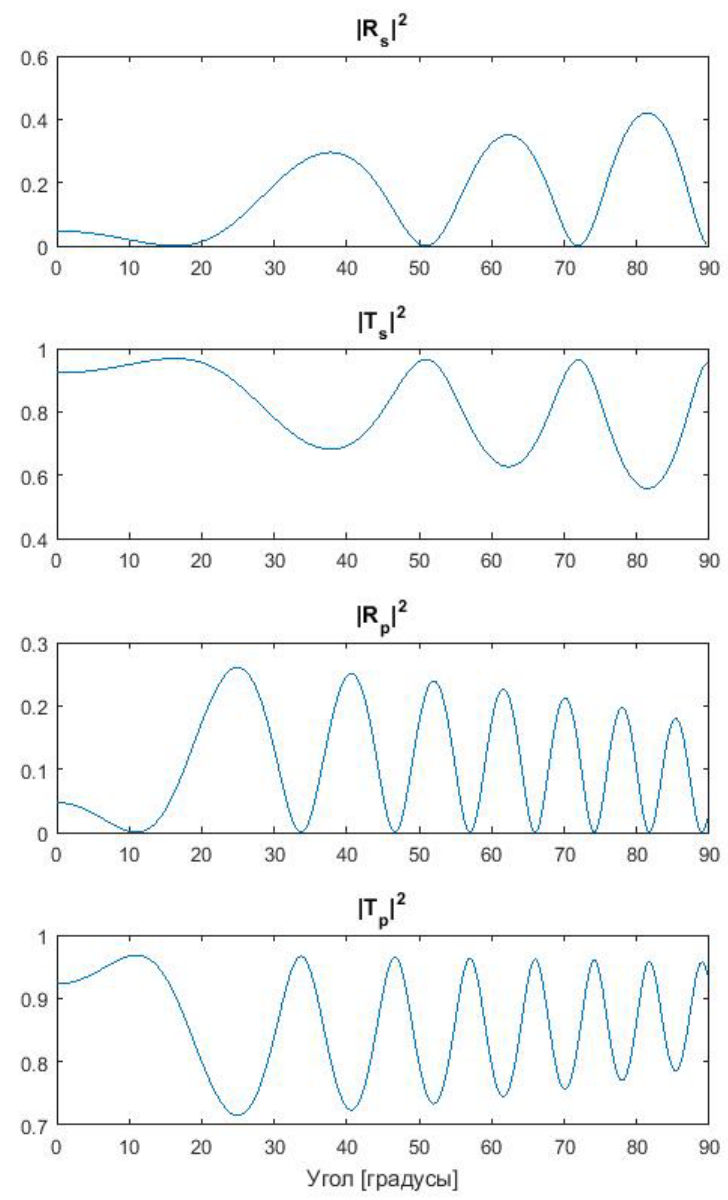

Fig. 2. The same angular diagrams as in Fig. 1, but for anisotropic ice at $\varepsilon_{22}=\varepsilon_{33}=3.18+i 0.0007, \varepsilon_{11}=1.5$ $+i 0.003$, the plate thickness is $320 \mathrm{~cm}$. 
In Fig. 3 shows the angular diagrams of the plate with a thickness reduced to $100 \mathrm{~cm}$.

If the thickness of the ice plates is $320 \mathrm{~cm}$, adding a layer of water to this plate leads to a decrease in transmission coefficients, without noticeable changes in the structure of the angular diagrams. The dielectric constant of water, calculated by the Debye formula [16] for frequency $1 \mathrm{GHz}$ at zero degrees Celsius, has the value $\varepsilon=86.78+9.14$ i. The real part is more than an order of magnitude higher than that of ice, and the imaginary part, which determines the damping of waves, exceeds it by more than 3 orders
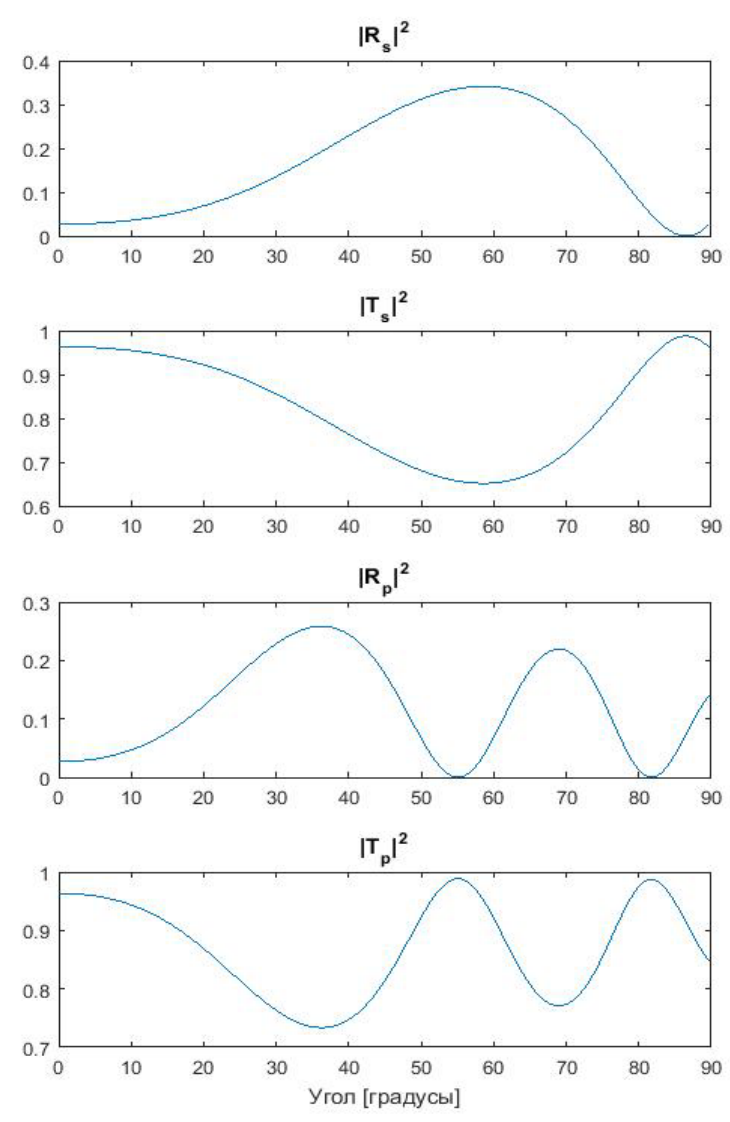

Fig. 3. The same angular diagrams as in Fig. 2 for anisotropic ice with a decrease in plate thickness up to $100 \mathrm{~cm}$.
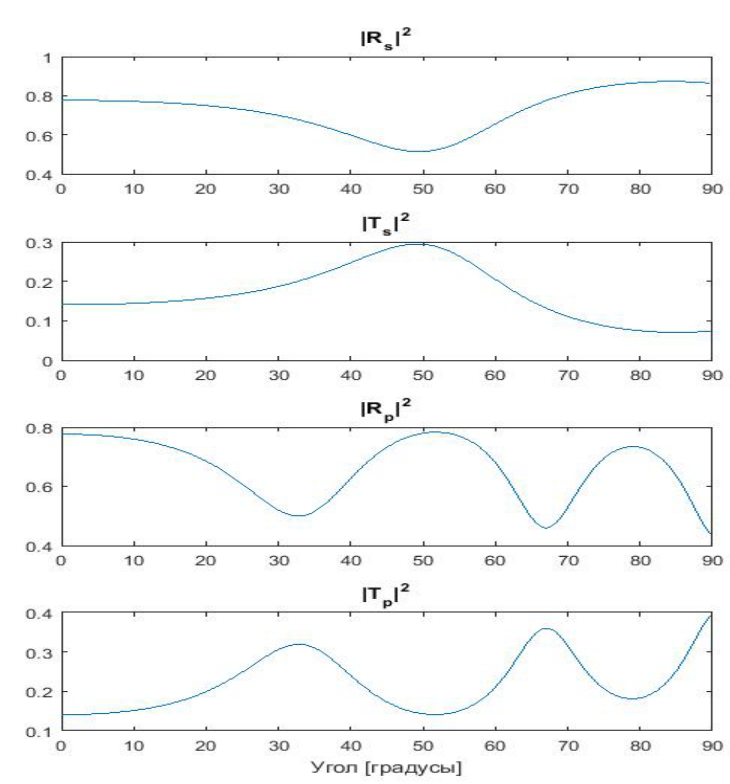

Fig. 4. The angular diagrams of an anisotropic ice plate, with the same parameters as in Fig. 3, but a layer of water $3 \mathrm{~mm}$ thick. was added to an ice sheet $100 \mathrm{~cm}$ thick.

of magnitude. In the event that the thickness of the plate is reduced to 100 $\mathrm{cm}$, then between the thin layer of water and the ice layer, resonance interactions that significantly change the behavior of the angular diagrams are possible. Fig. 4 shows the angular diagrams of an anisotropic ice plate, with the same parameters as in Fig. 3, but a layer of water $3 \mathrm{~mm}$ thick was added to the ice sheet $100 \mathrm{~cm}$ thick.

\section{CONCLUSION}

The identification of a class of quasianisotropic media expands the set of problems solvable with the help of Fresnel equations. Generalizations of the FresnelAiri equations for this class of media make it possible to study theoretical models of the passage of plane waves through planeparallel plates of anisotropic dielectrics, 
both transparent and absorbed, the plates can be ferromagnets magnetized to saturation $[9,10]$.

It can be assumed that the described methods are applicable to certain gyrotropic media and ferroelectrics. The class of quasi-anisotropic media is rather wide, but does not cover all the anisotropic media allowed by the Maxwell equations. It seems promising to apply the described generalizations of Fresnel's formulas to construct and study numerical stochastic models, following the example of [15]. There is a large number of recurrent and matrix approaches to the generalization of Fresnel's formulas to multilayer isotropic plates $[2,3]$. From the point of view of the author, the reduction of the FresnelAiry formulas for quasi-anisotropic media to the standard form of the linear equation (4) should facilitate studies of the problems of computational stability of the corresponding numerical algorithms. For media with wave attenuation, the matrix $\hat{U}$ is a matrix with a diagonal predominance and the standard methods for solving linear equations must be computationally stable.

The class of quasi-anisotropic media is much broader than the cases considered for the example, it includes all isotropic media, transparent and with absorption, and also some ferromagnetic $[9,10]$ and, possibly, gyrotropic media under certain conditions. Further studies must clarify the boundaries of this class and the conditions of applicability, for example, for ferroelectrics. It is known that the Fresnel equations may give incorrect results for for media with an amplification, in this case more complicated methods that take into account the causality relations in the propagation of waves are required [13].

\section{APPENDIX 1.}

Permissible tensors of Dielectric and MAGNETIC PERMEABILITIES FOR QUASIANISOTROPIC MEDIA

Let us write the tensors of the dielectric and magnetic permeabilities, admissible for anisotropic media. The symbol $\mathrm{X}$ denotes admissible components on which the properties of the corresponding waves do not depend.

\section{For s-waves}

$$
\mathbf{A}_{s}=\left(\begin{array}{c}
H_{x} \\
H_{y} \\
E_{z}
\end{array}\right) ; \hat{\boldsymbol{\varepsilon}}=\left(\begin{array}{ccc}
\times & \times & 0 \\
\times & \times & 0 \\
\times & \times & \varepsilon_{33}
\end{array}\right) ; \hat{\boldsymbol{\mu}}=\left(\begin{array}{ccc}
\mu_{11} & \mu_{12} & \times \\
\mu_{21} & \mu_{22} & \times \\
0 & 0 & \times
\end{array}\right) .
$$

\section{For p-waves}

$$
\mathbf{A}_{p}=\left(\begin{array}{c}
-E_{x} \\
-E_{y} \\
H_{z}
\end{array}\right) ; \widehat{\boldsymbol{\varepsilon}}=\left(\begin{array}{ccc}
\varepsilon_{11} & \varepsilon_{12} & \times \\
\varepsilon_{21} & \varepsilon_{22} & \times \\
0 & 0 & \times
\end{array}\right) ; \hat{\boldsymbol{\mu}}=\left(\begin{array}{ccc}
\times & \times & 0 \\
\times & \times & 0 \\
\times & \times & \mu_{33}
\end{array}\right) .
$$

For s- and p-waves simultaneously:

$$
\widehat{\boldsymbol{\varepsilon}}=\left(\begin{array}{ccc}
\varepsilon_{11} & \varepsilon_{12} & 0 \\
\varepsilon_{21} & \varepsilon_{22} & 0 \\
0 & 0 & \varepsilon_{33}
\end{array}\right) ; \hat{\boldsymbol{\mu}}=\left(\begin{array}{ccc}
\mu_{11} & \mu_{12} & 0 \\
\mu_{21} & \mu_{22} & 0 \\
0 & 0 & \mu_{33}
\end{array}\right) .
$$

If in the medium permeability tensors there are no cross terms with respect to $z$, then for such media it is possible to calculate the Fresnel coefficients for waves with $s$ - and $p$-polarizations at $k_{z}=0$ and given $k_{y}$. 


\section{APPENDIX 2.}

FRESNEL COEFFICIENTS FOR QUASIANISOTROPIC MEDIA

$$
\begin{aligned}
& \mu(1, u, m, n)=\mu_{m, n,[u]} ; \mu(-1, u, m, n)=\varepsilon_{m, n,[u]} \\
& D_{3}(e, u)=(w / c)(\mu(e, u, 1,1) \mu(e, u, 2,2)- \\
& -\mu(e, u, 1,2) \mu(e, u, 2,1)) ; \\
& k_{1, e,[u]}=-k_{y} \frac{\mu(e, u, 1,2)+\mu(e, u, 2,1)}{\mu(e, u, 1,1)} ; \\
& k_{2, e,[u]}=\sqrt{\left(k_{1, e,[u]}\right)^{2}-\frac{\mu(e, u, 2,2)}{\mu(e, u, 1,1)} k_{y}^{2}+\frac{\omega \mu(-e, u, 3,3)}{c \mu(e, u, 1,1)} D_{3}(e, u)} \\
& k_{x}(e, q, u)=k_{1, e,[u]}+q k_{2, e,[u]} ; \\
& D_{2}(e, q, u)=-\left(k_{x}(e, q, u) \mu(e, u, 1,1)+k_{2} \mu(e, u, 2,1)\right) ; \\
& F(e, q, u)=\frac{D_{2}(e, q, u)}{D_{3}(e, u)} ; \\
& T(e, q, u)=1+R(e, q, u) ; \\
& R(e, q, u)=\frac{F(e, q, u)-F(e, q, u+q)}{F(e, q, u+q)-F(e,-q, u)} ; \\
& \beta(e, q, u)=\exp \left(i k_{x[u]}(e, q, u) q d_{[u]}\right) .
\end{aligned}
$$

\section{REFERENCES}

1. Landau LD, Lifshitz EM. Theoretical physics, vol. VIII. Electrodynamics of continuous media. Moscow, Fizmatlit Publ., 2003, 656 p.

2. Born M., Wolf E. Principles of Optics. London, Pergamon Press, 1959, 720 p.

3. Rosenberg GW. Optika tonkosloynykh pokrytiy [Optics of thin-layer coatings]. Moscow, Fizmatlit Publ., 1958, 570 p.

4. Brekhovskih LM. Volny v sloistykh sredakh [Waves in layered media]. Moscow, Nauka Publ., 1973, 343 p.

5. Gorshkov MM. Ellipsometriya [Ellipsometry]. Moscow, Sovetskoe radio Publ., 1974, 200 p.
6. Shutko AM. SVCH-radiometriya vodnoy poverkhnosti i pochvogruntov [Microwave radiometry of water and ground surfaces]. Moscow, Nauka Publ., 1986, 188p.

7. Vashkovskii VA, Stalmakhov VS, Sharaevskii YuP. Magnitostaticheskie volny $v$ elektronike sverkhyysokikh chastot [Magnetostatic waves in electronics of ultrahigh frequencies]. Saratov, University Publ. House, 1993, 311 p.

8. Bespyatykh YuI, Bugaev AS, Dikstein IE. Surface polaritons in composite media with temporal dispersion of dielectric and magnetic permeabilities. FTT， 2001， 43(11):2043-2047 (in Russ.).

9. Evtikhov MG. The Snellius and Fresnel ratios for electromagnetic waves with constant linear polarization. Journal of Communications Technology and Electronics, 2010, 55(8):915-922.

10.Evtikhov MG, Nikitov SA, Novichikhin EP. Teoreticheskoe issledovanie spektrov TE voln $v$ mnogosloynikh plenkakh iz ferromagnitnykh $i$ dielektricheskikh materialov [Theoretical study of spectra of TE waves in multilayer films of ferromagnetic and dielectric materials. Saratov, University Publ. House, 2013, $68 \mathrm{p}$.

11.Evtikhov MG. Predelno razrezhennye matritsy [Extremely sparse matrices]. Radioelektronika.

Nanosistemy. Informatsionnye Technologii (RENSIT), 2011, 3(1):97-101 (in Russ.). 
12.Evtikhov MG. Ekstensivnoe umnozhenie [Extensive multiplication]. RENSIT, 2013, 5(1):143-151 (in Russ.).

13. Kolokolov AA. Formuly Frenelya i princip prichinnosti [Fresnel formulas and the causality principle]. UFN, 1999, 169:1025 (in Russ.).

14. Basharinov AE, Tuchkov LT, Polyakov VM, Ananov NI. Izmerenie radioteplovykh $i$ plazmennykh iqlucheniy [Measurement of radiothermal and plasma radiation]. Moscow, Sovetskoe Radio Publ., 1968, $390 \mathrm{p}$.

15.Evtikhov MG. Primenenie modeli Frenelya-Eyri pri issledovanii rezonansnykh ostzillyatsiy ploskikh electromagnitnykh voln [Application of the Fresnel-Airy model in the study of resonant oscillations of plane electromagnetic waves]. Zhurnal radioelektroniki [ Journal of Radioelectronics], 2017, 9:1684-1719 (in Russ.), http:// jre.cplire.ru/jre/sep17/11/text.pdf accessed 27 September 2017.

16.Uzlov VA, Shishkov GI, Shcherbakov VV. Osnovnye fizicheskie parametry snezhnogo pokrova [The main physical parameters of the snow cover]. Proceedings of R.E. Alekseev Nizhny Novgorod State Technical University, 2014, 103(1):119-129 (in Russ.). 